\title{
Thick-wall, Liquid-Filled Quartz Capillaries for Scintillation and Wavelength Shifting Applications
}

\author{
R. Ruchti, ${ }^{1}$ B. Baumbaugh, N. Dev, B. Dolezal, C. Jessop, C. Mohs, N. Siwietz, J. \\ Taylor, J. Twaddle and M. Vigneault \\ University of Notre Dame \\ Notre Dame, Indiana 46556 USA \\ E-mail: rruchti@nd.edu, ckellyhavens@yahoo.com, Nabarun.Dev.1@nd.edu, \\ bdolezal@saintjoehigh.com, cjessop@nd.edu, nikemohs@gmail.com, \\ njsiwietz@gmail.com, jtaylor@elkhart.k12.in.us, jtwaddle1@gmail.com, \\ Mark.j.vigneault.1@nd.edu
}

\begin{abstract}
We have been developing a readout method for Shashlik Electromagnetic Calorimetry based upon liquid-filled capillaries fabricated from radiation hard (high $\mathrm{OH}$ content) fused silica. The liquids are waveshifters, that currently shift scintillation light from $425 \mathrm{~nm}$, the emission from LYSO $(\mathrm{Ce})$ crystals to wavelengths in the green $(500 \mathrm{~nm})$. While the liquid in the capillary core serves as the light source, the thick-wall of the Quartz capillaries provide the bulk of the optical path through which the wave shifted light is transmitted to photosensors. Characteristics and performance of prototype capillaries are presented
\end{abstract}

38th International Conference on High Energy Physics

3-10 August 2016

Chicago, USA

${ }^{1}$ Speaker 


\section{Introduction}

Calorimeters for use in high flux and high radiation experimental conditions (for example in hadron collider experiments at the LHC) are expected to suffer significant radiation damage during the HL-LHC era and beyond. To address these challenges, we have been focusing our attention on radiation tolerant Shashlik-type EM calorimeters of very compact design with a structure consisting of alternating layers of very dense absorber $(2.5 \mathrm{~mm}$ thick $\mathrm{W}$ plates $)$ and high efficiency scintillator(1.5mm thick LYSO plates). [1-3] This combination of materials affords a dense, compact design, with small Moliere Radius $(13.7 \mathrm{~mm})$ and short physical length (135 mm) corresponding to $\sim 24$ radiation lengths (Xo) in depth. The high brightness of the LYSO and short optical path lengths allow for a detector design that is both robust against radiation damage and event pile up, both central issues for detector operation at high luminosity. The LYSO and tungsten layers each have five machined holes, four for readout of the detector via WLS capillaries, and one in the center for a calibration fiber. In Fig. 1 (Right), four capillaries penetrate through the length of the Shashlik module to sample the scintillation light produced in the LYSO crystals.

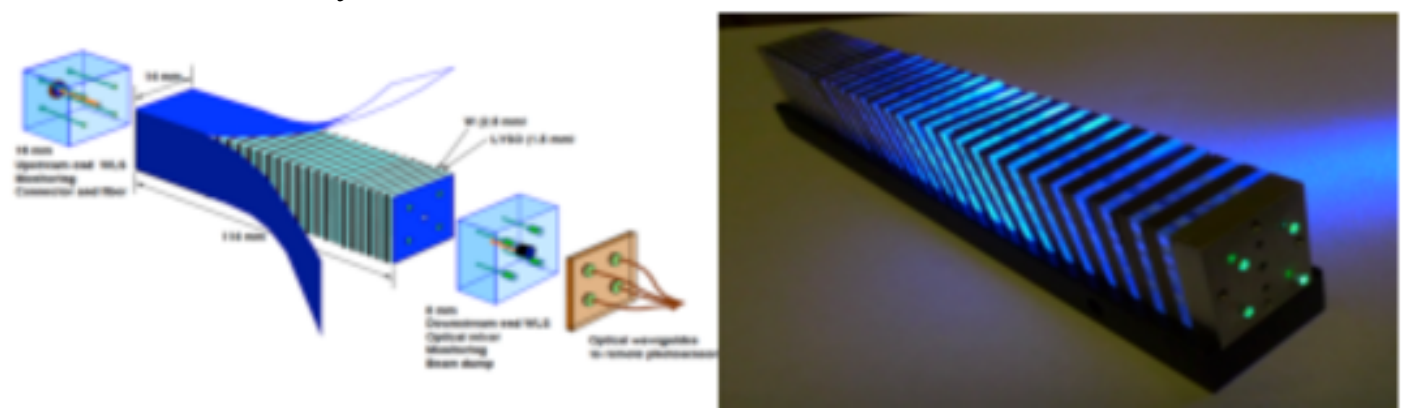

Figure 1. Left: A Shashlik module based upon interleaved W and LYSO(Ce) layers. Four WLS liquid-filled quartz capillaries are used to detect the scintillation light and transfer the light to photosensors at the downstream end of the module (right hand side in the figure). Right: An assembled Shashlik Module.

Our choice of capillary material is high $\mathrm{OH}$ content fused silica, [4] selected for its radiation hardness, and based upon prior experience from the CMS forward hadron calorimeter (HF). The capillary cross-sectional dimensions are: outer diameter of $1 \mathrm{~mm}$ and core diameter of $0.4 \mathrm{~mm}$, determined from a SLitrani simulation study. The capillary lengths are $185 \mathrm{~mm}$, of which $114 \mathrm{~mm}$ lies within the active volume of the Shashlik module itself. The liquid waveshifters under study are based on a quenched EJ309 liquid base, and containing the fluorescent dye DSB1. [5] The fluorescence characteristics of DSB1 are similar to the wellknown Y11, absorbing light near 425nm and emitting light near 500nm, but DSB1 has a faster response by a factor of $\sim 2$. The refractive indices of the core liquid and quartz are 1.57 and 1.46 respectively. The quartz capillary itself has no external cladding other than an air layer. The light traveling within the liquid core is prevented from reaching the readout directly by a ruby quartz "core block", whereas light from the quartz/liquid combination is not. Light propagation solely within the WLS liquid suffers from two issues: self-absorption of light due to the WLS 
dye itself; and possible damage to WLS liquid medium due to irradiation effects. Both can in principle lead to longitudinal non-uniformity of light collection. However since the WLS liquid occupies only $16 \%$ of the volume of the capillary, the optical transmission of the WLS light occurs predominantly in the rad hard quartz material, helping to level the longitudinal response of the system. Fig. 3 (Left) provides a schematic of the light collection and transmission process and Fig. 3 (Right) shows images of fabricated capillaries.
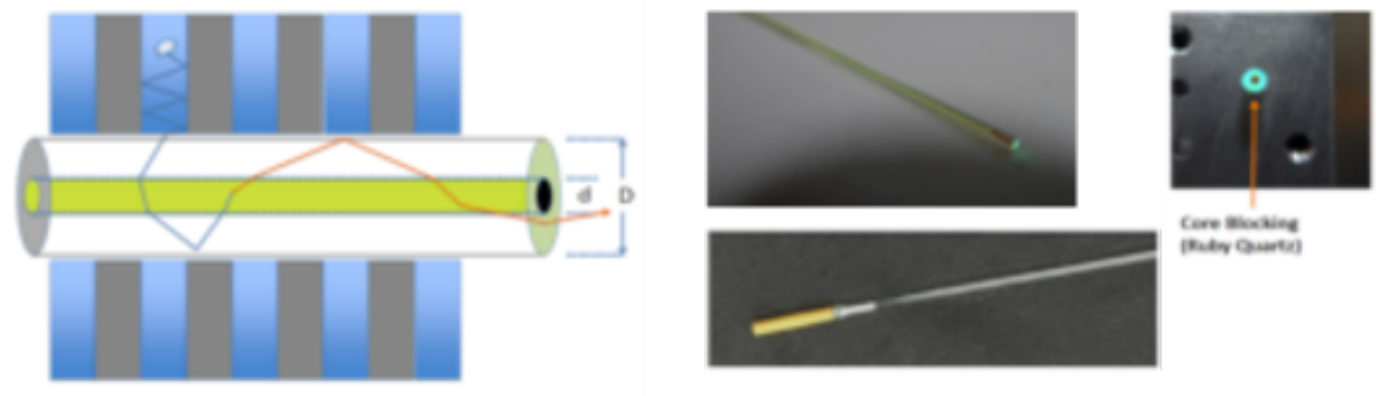

Figure 3. Left: A schematic of the light collection approach. Right: Photos of the capillary structure.

\section{R\&D Program}

The objectives of the program are to: optimize the capillary structure for efficiency and uniformity of light collection; and measure performance of the capillaries as readout elements both before and after irradiation. The capillary fabrication procedure involves the following steps (refer to Fig.3). First, a spherical reservoir bubble is blown into the capillary that serves as an expansion/contraction reservoir for the WLS fluid. Second, a ruby quartz "plug" of $3.5 \mathrm{~mm}$ length is inserted and thermally fused into the end of the capillary opposite to the reservoir bubble. This end is then ground to a high optical finish and serves as the readout end of the structure. Third, the open end near the reservoir is attached to a vacuum system, which evacuates the capillary. Fourth the capillary is then filled with the WLS fluid. Lastly, the capillary is then sealed either by fusion sealing or epoxy sealing. The capillary is then subjected to quality control testing, by excitation through the quartz sidewall with an LED of wavelength $\lambda=425 \mathrm{~nm}$, and measuring the amount of light collected at the readout end by means of a $\mathrm{P} / \mathrm{N}$ diode. This allows measurement of the overall light level and optical attenuation length. An example of such a measurement is shown in Fig. 5, with the gray data points indicating the capillary light collection behavior just after fabrication. Next, a $4 \mathrm{~mm}$-wide thin overcoat (band) of $\mathrm{TiO} 2$ paint is applied extramurally to the capillary near the reservoir end (refer to Fig. 3 lower right). As the red data points in Fig. 5 (Left) show, the TiO2 banding (a Lambertian reflector) improves the light collection from locations furthest from the readout end, but also benefits light collection overall. Lastly, the capillary is exposed to $50 \mathrm{Mrad}$ dose of gamma radiation from a 60Co source at the Notre Dame Radiation Chemistry Laboratory. As shown by the green data points in Figure 5, two remarkable effects are observed: the overall light level rises from the middle to the far end due to a shift in the optical absorption spectrum of the WLS liquid (Fig. 5 Right); and the slope in the optical attenuation at the near (readout) end is suppressed, due to radiation darkening of the ruby quartz core blocking. Under further doses of radiation in 
50Mrad steps, the overall light level from the capillary drops, but in a fashion consistent with an overall scale factor of about $\sim 25 \%$ in light reduction per $50 \mathrm{Mrad}$ of dose up to $200 \mathrm{Mrad}$ of total dose, the limit of current measurements. For reference, $150 \mathrm{Mrad}$ is the maximum total ionization dose expected in CMS endocarps near $\eta=3$ for integrated luminosity of $3000 \mathrm{fb}-1$. [1] Capillaries of the type described have been used successfully in a recent beam test of a $4 \times 4$ array of Shashlik Modules at CERN and reported at this conference. [1] Those results and these here indicate that liquid-filled WLS Quartz capillaries can provide an efficient and radiation hard readout technique for light-based calorimetry in high radiation environments.
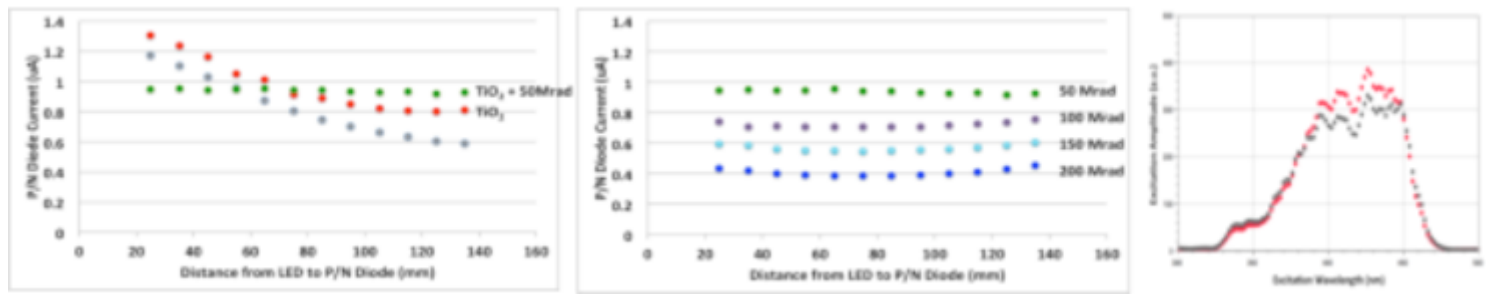

Figure 5. Left: Measured light level in a $\mathrm{P} / \mathrm{N}$ diode at the readout end of a quartz capillary as a function of the longitudinal distance from the diode to the exciting light from an LED positioned to inject its light though the sidewall of the capillary. (1) Gray data points: measured after initial fabrication and unirradiated. (2) Red data points: After TiO2 sidewall band coating at the reservoir end near the $160 \mathrm{~mm}$ position and unirradiated. (3) Green data points: after exposure to 50Mrad of gamma irradiation. Center: Continued measurements after additional levels of gamma irradiation. Right: Excitation spectrum of the DSB1 liquid waveshifter before (gray dots) and after (red dots) $50 \mathrm{Mrad}$ of gamma irradiation. These spectra correspond to WLS emission at 500nm. Measurement using a Varian Cary Eclipse Fluorescence Spectrometer.

\section{Acknowledgements}

We thank the Notre Dame Radiation Chemistry Laboratory for the services of their glass shop and radiation facilities and the Notre Dame QuarkNet Center for teacher and student support. We thank the CERN Laboratory, the CMS Experiment, the USCMS Project Office and our colleagues in the CMS Shashlik Working Group [6] for their continued support. And we thank the US National Science Foundation, the US Department of Energy, and University of Notre Dame for their funding support of this program of research and development.

\section{References}

[1] B. Cox, et al, The Shashlik Calorimeter, a LYSO/W plate Calorimeter for Precision EM Calorimetry in the High Luminosity LHC environment, paper presented at this conference.

[2] R. Ruchti, et al, High Performance, Radiation Hard Light-Based EM Calorimetry for Particle Physics Applications, CPAD Instrumentation Frontier Meeting, 5-7 October 2015, University of Texas at Arlington. https://indico.hep.anl.gov/indico/conferenceOtherViews.py? view $=$ standard $\&$ confId $=625$

[3] Ren-Yuan Zhu, et al, A Crystal Shashlik Electromagnetic Calorimeter for Future HEP Experiments, paper presented at CALOR 2016, Daegu, Republic of Korea, 15-20 May 2016. https://indico.cern.ch/event/472938/contributions/1150709/attachments/1273761/1888812/ryz_1605 17_calor16_Shashlik.pdf 
[4] Capillaries tubes are produced by Polymicro Technologies, Molex, LLC, Phoenix, AZ.

[5] DSB1 is a commercially available organic fluorescent dye and EJ309 is a scintillating liquid base developed by Eljen Technology, Inc, Sweetwater, TX.

[6] https://www3.nd.edu/ rruchti/CMS_Shashlik_Collaboration.pdf 